\title{
A simple size-tailored algorithm for the removal of chest drain following minimally invasive lobectomy: a prospective randomized study
}

\author{
Davor Stamenovic ${ }^{1,2}$ - Michael Dusmet ${ }^{3} \cdot$ Thomas Schneider $^{1} \cdot$ Eric Roessner $^{2} \cdot$ Antje Messerschmidt $^{1,4}$
}

Received: 26 August 2021 / Accepted: 21 November 2021 / Published online: 30 November 2021

(C) The Author(s) 2021

\begin{abstract}
Background The pleural space can resorb $0.11-0.36 \mathrm{ml} / \mathrm{kg}$ of body weight/hour (h) per hemithorax. There are only a limited number of studies on thresholds for chest drain removal (CDR) and all are based on arbitrary amounts, for example, $300 \mathrm{ml} /$ day. We studied an individualized size-based threshold for CDR-specifically $5 \mathrm{ml} / \mathrm{kg}$, a simple, easily applicable measure. Methods This is a single-center prospective randomized trial enrolling 80 patients undergoing VATS lobectomy. There were two groups: an experimental (E) group, in which once the daily output went down to $5 \mathrm{ml} / \mathrm{kg}$ the chest drain was removed and a control (C) group, with chest drain removal as per our current practice of less than $250 \mathrm{ml} / \mathrm{day}$.

Results The groups did not differ in pre- and peri- and postoperative characteristics, except for chest drain duration (mean, SD $2.02 \pm 0.97$ vs. $3.25 \pm 1.39$ days, $p<0.001$ ) and length of hospital stay (median, IQR $4.5 ; 3$ vs. 6 ; 2.75 days, $p=0.008$ ) in favor of $\mathrm{E}$ group. The re-intervention rate was the same in both groups (once in each group).

Conclusion The new threshold for chest drain removal following thoracoscopic lobectomy of $5 \mathrm{ml} / \mathrm{kg} / \mathrm{d}$ leads to both shorter chest drainage and hospital stay without apparent increase in morbidity. (Clinical registration number: DRKS00014252).
\end{abstract}

Keywords Chest drain · VATS lobectomy $\cdot$ Minimally invasive surgery $\cdot$ Anatomical lung resection · Daily output

The pleural space normally contains $0.13 \pm 0.60 \mathrm{ml} / \mathrm{kg}$ of hypo-oncotic fluid (mixture of microvascular filtrates and protein at $1 \mathrm{~g} / \mathrm{dl}$ ) [1,2]. The pleural fluid is produced by the parietal pleura, mainly in the less dependent parts, while absorption is by its lymphatic vessels, as well as the lymphatics of the mediastinum and diaphragm, mainly in the most dependent parts of the pleural space, maintaining an equilibrium at $0.01-0.02 \mathrm{ml} / \mathrm{kg} / \mathrm{h} \mathrm{[3]}$. However, if a pathological process is present (tumor involvement, inflammation, postoperative status after thoracic surgery), the effusion may come from visceral pleura, diaphragm, and peritoneum as well [4]. A tenfold increase in pleural fluid production only

\footnotetext{
Davor Stamenovic

davor.stamenovic@unimedizin-mainz.de

Vidia Kliniken Karlsruhe, Karlsruhe, Germany

University Thoracic Center Mainz, Mainz, Germany

Royal Brompton Hospital, London, UK

4 GFO Kliniken, Bonn, Germany
}

leads to a $15-20 \%$ increase in the volume of the pleural effusion [5].

Applying this to a patient weighing $70 \mathrm{~kg}$, approximately $470 \mathrm{ml}$ of effusion will be produced and absorbed daily in the pleural space or approximately $0.11-0.36 \mathrm{ml} / \mathrm{kg}$ of body weight/h per hemithorax $[6,7]$.

At present most decisions regarding CDR are arbitrary and vary widely between institutions both nationally and internationally. In Germany, these typically range between 100 and $300 \mathrm{ml} / \mathrm{d}$ [8], while in Denmark this seems to differ with an accepted threshold of up to $400 \mathrm{ml} / \mathrm{d}[9,10]$.

There are a limited number of publications available on the criteria for chest drain removal (CDR) after videoassisted thoracoscopic (VATS) lobectomy based on the output through the chest drain (CD), only one of which was a prospective randomized controlled study [11]. However, there are currently no prospective randomized studies of the fluid output per $24 \mathrm{~h}$ for CDR removal after VATS lobectomy based on the patient's size. 


\section{Objectives}

The primary objective is to examine a feasibility and safety of an algorithm with CDR once the daily output goes down to $5 \mathrm{ml} / \mathrm{kg} /$ day.

There were two primary endpoints of this study: the chest drainage duration (CDD) and the number of reinterventions caused by inadequate drainage of the pleural space.

The incidence of re-interventions (RI) will be used to calculate the number of the patients needed to be enrolled in a non-inferiority study aiming to reach 0.8 power for the maximum of $10 \%$ higher re-interventions rate in the experimental group compared with the active control group $(250 \mathrm{ml} / \mathrm{d})$.

The secondary endpoint is the rate of complications in both groups.

\section{Materials and methods}

This is a single-center prospective randomized pilot trial registered at the Germany register for clinical studies (DRKS00014252) and approved by the ethical committee of University of Heidelberg (S-159/2018). The study was conducted in accordance with the Declaration of Helsinki, and written informed consent was obtained from all patients.

Based on the historical data from our institution on successive 148 patients, who have undergone VATS lobectomy and a standard deviation derived from the CDD $(\mathrm{SD}=1.475), 72$ patients (36 in each group) are required for the study to achieve a power of 0.8 specifically for the one-day difference in CDD between the groups.

\section{Randomization}

The numbers 1 to 100 are placed in an arbitrary order using an Excel random number generator. An independent, non-medical person transfers these numbers onto a sheet of paper. These sheets are then placed in envelopes numbered 1 to 100 and sealed. (Example: envelope number 5 contains number 40).

Once the eligibility criteria have been met, an envelope corresponding to the particular patient (the fifth envelope belongs to the 5 th enrolled patient, 10th to the 10th, etc.) is unsealed and a number within revealed. Should an even number appear, that patient belonged to the experimental group. Patients with odd numbers went into the control group.

Eighty patients undergoing VATS lobectomy were randomized to the two groups: the experimental (E) group, with CDR once the output dropped to $5 \mathrm{ml} / \mathrm{kg} / \mathrm{d}$ and a control (C) group, with CDR at less than $250 \mathrm{ml} / \mathrm{d}$.

All patients over 18 years of age, capable of giving consent and undergoing a minimally invasive lobectomy (for benign disease, primary lung cancer, or a pulmonary metastasis from another primary tumor), were eligible for the study. The diagnosis of the lesion could be obtained pre or intraoperatively.

Exclusion criteria were any other type of resection or utilization of an alternative approach (including conversion thoracotomies), pleural carcinosis, any air leak, or revision surgery within the first $24 \mathrm{~h}$ postoperatively (for example for bleeding), heart or kidney failure, as well as BMI $>30 \mathrm{~kg} /$ $\mathrm{m}^{2}$ or $<18 \mathrm{~kg} / \mathrm{m}^{2}$.

\section{Operative management}

VATS lobectomy with systematic lymph node dissection was performed by means of three, two, or single port incisions at the surgeon's discretion. A single chest drain (24 F, Rocket Medical, Washington, DC, USA) was placed in the pleural space at the end of the procedure in every case.

On the 1st postoperative day, clinical data were recorded followed by randomization of the patient into the allocated treatment group provided there was not an on-going air leak.

The postoperative care of the patients was standardized with at least daily visits starting on postoperative day (POD) 1 in the intermediate care unit (IMC), as well as on the ward until discharge. Fluid output was recorded daily by the nursing staff at $7 \mathrm{am}$. Chest X-rays (PA and lateral) were performed the day after CDR and were reviewed by two physicians (one of which was the patient's surgeon). If the patient was well and the $\mathrm{X}$-rays showed no complications the patient was in principle discharged home.

The patients were monitored daily while in hospital and for 2 weeks following discharge. All complications were recorded, specifically the need for $\mathrm{CD}$ reinsertion or for thoracentesis or the development of pleural empyema or any need of antibiotic treatment for postoperative pneumonia. Complications were defined according to the definitions from the Society of Thoracic Surgeons and The European Society of Thoracic Surgeons [12].

Patients were instructed to seek for help from our department, either directly at the outpatient clinic, emergency room, or by calling, in particular if they had any respiratory problems.

The patients were reviewed in the outpatient clinic at approximately two weeks after discharge. In addition to clinical (e.g., new dyspnea) and/or radiological findings, an ultrasound examination was routinely carried out. Reintervention (thoracentesis, CD reinsertion, or thoracoscopy) was carried out if there was a pleural effusion of more than $500 \mathrm{ml}$ and if the patient complained of dyspnea: The risks 
of the evacuation of a pleural effusion of less than $500 \mathrm{ml}$ usually outweigh the benefits [13] and these small effusions are sometimes difficult to visualize with an X-ray [14].

The amount of effusion was determined by means of an ultrasound examination: The sum of the basal distance between the lungs, the diaphragm, and the lateral height of the effusion multiplied by 70 gives a good estimate of the volume of the effusion $(r=0.87)[15,16]$.

\section{Statistical analysis}

All but one of the continuous variables were normally distributed and therefore compared by means of Student's t test. The single continuous non-normally distributed variable was the length of hospital stay and Wilcoxon's test was utilized for comparison. Categorical variables were analyzed using
Fisher's test in case of two variables and Wald test if more than two needed to be analyzed.

Data were analyzed using R stats [17], Microsoft Excel@, and G-Power [18].

\section{Results}

From April 2018 to December 2019, 87 patients underwent randomization after fulfilling the entry criteria. Seven patients were excluded after randomization (Fig. 1) [19]. All of them were men: one patient developed an air leak on the 2 nd postoperative day, four were diagnosed with heart failure (BNP > 15.000), one was excluded due to a breach of the study protocol (violation of the time of CDR), and

Fig. 1 CONSORT flow diagram

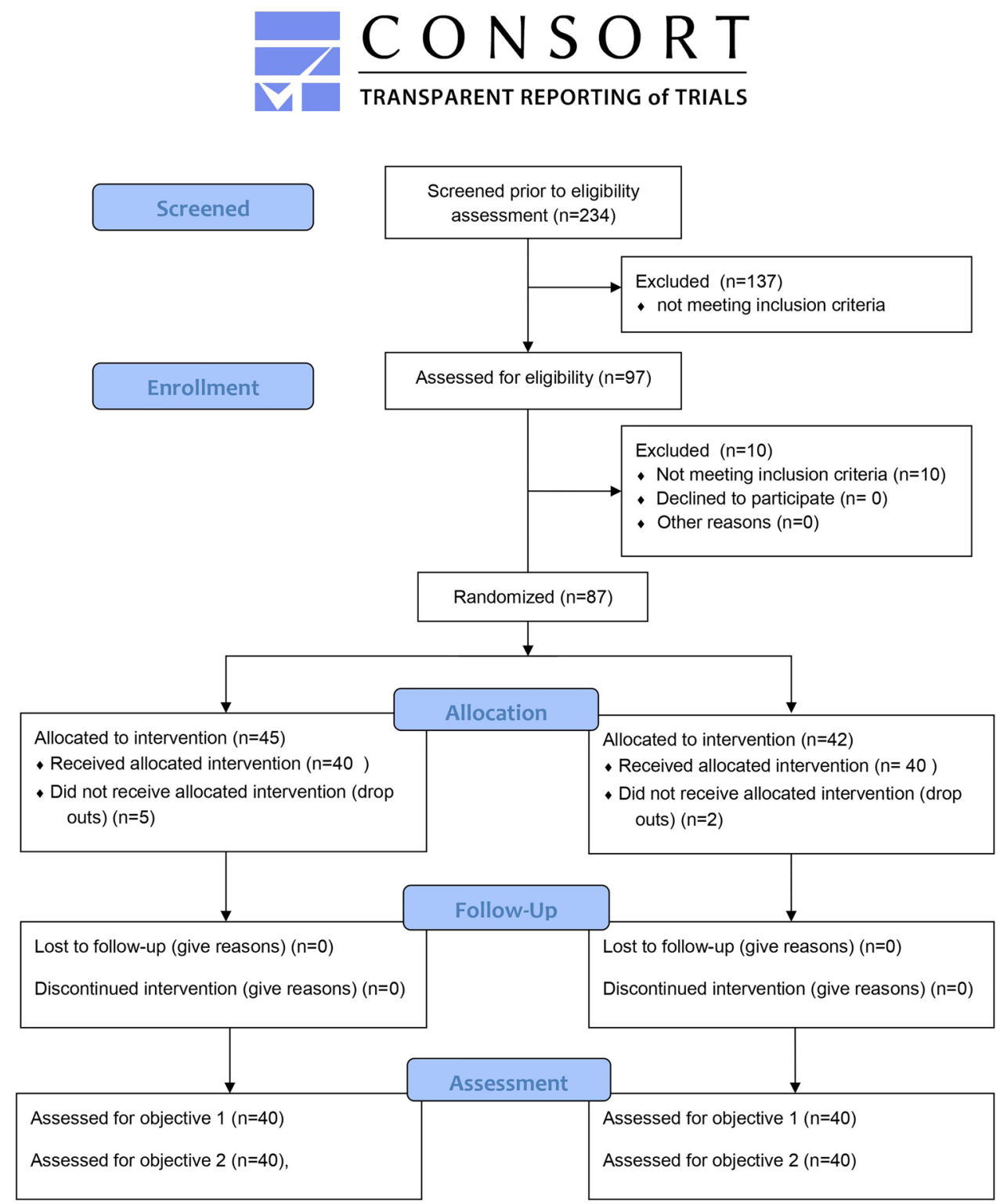


Table 1 Demographic characteristics of cohorts

\begin{tabular}{llll}
\hline Characteristic & Group E $(n=40)$ & Group C $(n=40)$ & $p$ \\
\hline Age (mean, SD) & $66(9)$ & $68(12)$ & 0.53 \\
Sex (male, percentage) & $28(70 \%)$ & $25(62.5 \%)$ & 0.64 \\
FEV1 (mean, SD) & $0.82(0.24)$ & $0.84(0.18)$ & 0.67 \\
DLCO (mean, SD) & $0.7(0.23)$ & $0.73(0.19)$ & 0.54 \\
ASA score (median, & $3(2-4)$ & $3(2-4)$ & 0.46 \\
$\quad$ range) & & & \\
BMI (mean, SD) & 25.2 & 24 & 0.25 \\
Preoperative CT (\%) & $5(12.5 \%)$ & $6(15 \%)$ & 1 \\
Actual smoker (\%) & $11(27.5)$ & $15(37.5 \%)$ & 0.47 \\
\hline
\end{tabular}

$E$ experimental; $C$ control; $S D$ standard deviation; $F E V_{1}$ forced expiratory volume in $1 \mathrm{~s} ; D L C O$ diffusing capacity; $A S A$ American Society of Anesthesiologists; $B M I$ body mass index; $C T$ chemotherapy

one developed pneumonia, bronchopleural fistula (BPF), and pleural empyema.

The two groups were very similar, as shown (Table 1).

The perioperative characteristics of the two patient groups were very similar (Table 2; Fig. 2). We also looked at how much adhesiolysis was required. This varied from none, around one lobe, and around more than one lobe and this was not significantly different between the two groups $(p=0.75)$. We also looked at the underlying disease process (benign disease vs primary lung cancer vs metastatic disease) and again there were no significant differences between the two groups $(p=0.81)$.

The difference in CDD of 1.22 days $(29.4 \mathrm{~h})$ in favor of the experimental treatment is highly significant $(\delta=1.02011)$. Both the upper limit of the $95 \%$ CI $(-0.6894)$ which is lower than 0 and the results of $t$ test $(\mathrm{t}(69.81)=-4.562, p=0.001$ show superiority of the experimental treatment for CDD compared to the control treatment. The power of this results, as the probability of rejecting a false Ho is very high at 0.99 .

There was one re-intervention in each group: in the $\mathrm{C}$ group, an 86-year-old female patient underwent a reinsertion of the $\mathrm{CD}$ on the eighth postoperative day (POD) the fourth day after CDR-due to a parapneumonic effusion. In the E group an 81-year-old female patient was re-drained on the second postoperative day-a day after CDR occurred for symptomatic pleural effusion.
Table 2 Perioperative characteristics of cohorts

\begin{tabular}{lccc}
\hline Characteristic & Group E $(n=40)$ & Group C $(n=40)$ & $p$ \\
\hline OP length, min (mean, SD) & $162(35)$ & $155(38)$ & 0.39 \\
pT, cm (SD) & $3.1(1.4)$ & $2.9(1.5)$ & 0.47 \\
Nr. LN removed (SD) & $18(7)$ & $21(8)$ & 0.2 \\
Complications (\%) & $7(17.5)$ & $9(22.5)$ & 0.32 \\
Chest drain duration, days (mean, SD) & $2.02(0.97)$ & $3.25(1.39)$ & 0.000 \\
LOS, days (median, IQR) & $4.5(3)$ & $6(2.75)$ & 0.008 \\
Nr. of re-intervention (\%) & $1(2.5)$ & $1(2.5)$ & 1 \\
\hline
\end{tabular}

$E$ experimental; $A C$ active control; $O P$ operation; $p T$ pathological tumor size; $S D$ standard deviation; $N r$. number; $L N$ lymphatic nodes; $L O S$ length of hospital stay; IQR interquartile range

Fig. 2 Lobectomy by groups. $L L L$ left lower lobectomy; $L U L$ left upper lobectomy; $M L$ middle lobe lobectomy; $R L L$ right lower lobectomy; $R U L$ right upper lobectomy; $C$ control group; $E$ experimental group

\section{Lobectomy type by groups}

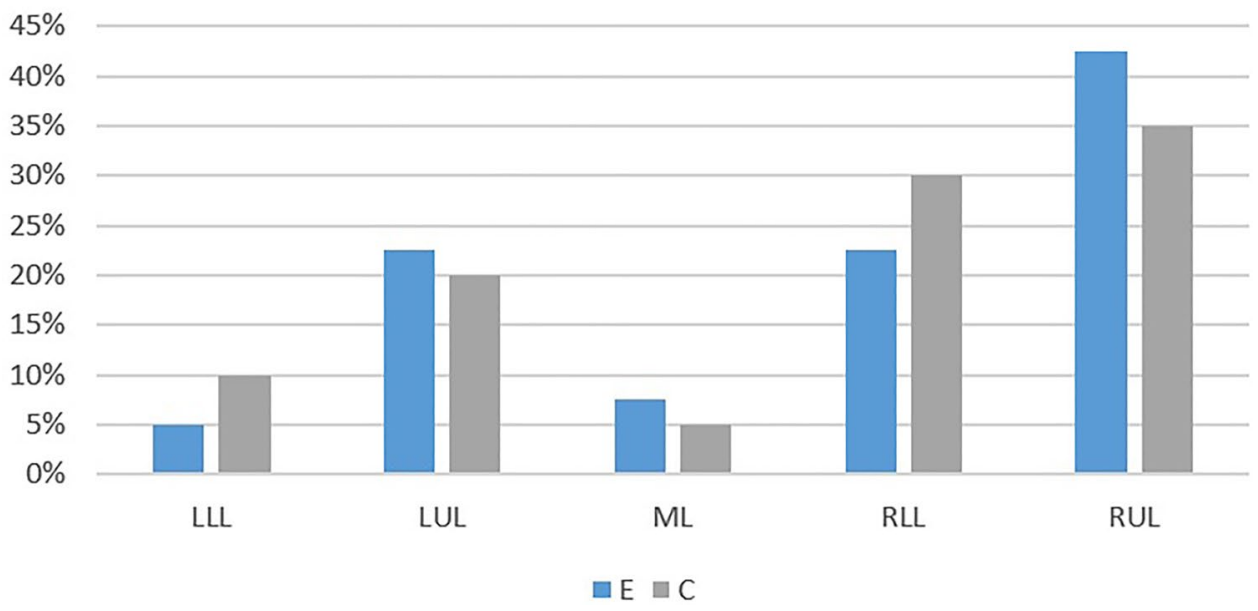


However as this study was not powered to study the rate of re-intervention (this would have required 280 patients) this result is not conclusive.

Apart from these two re-interventions, there was another case with pleural effusion, belonging to the $\mathrm{C}$ group, at the routine postoperative control at outpatient clinic two weeks after the patient was discharged. Despite the amount of pleural effusion $(500 \mathrm{ml})$, the patient was asymptomatic and thus no intervention was performed.

The complication rate was comparable and nonsignificantly higher in the $\mathrm{C}$ group with a higher incidence of pneumonia than in the E group (4 vs. 2) as shown in Table 3. There were more cases of atrial fibrillation in the $\mathrm{E}$ group ( 3 vs 1$)$. The calculated relative risk of having complications in the control group was 1.286 compared to the E group.

As mentioned, one patient (group C) developed a BPF and was excluded from the analysis. There were no deaths in either study group (either in hospital or during the 2 weeks of follow-up).

Finally, four patients were readmitted within first two weeks after discharge from hospital: three in group $\mathrm{C}$ and one in group $\mathrm{E}$.

Control group: one patient was readmitted on day 4 postdischarge to drain a pneumothorax (no effusion present). Another patient was readmitted 2 days post-discharge with respiratory failure (exacerbation of COPD). The 3rd patient was readmitted day 7 post-discharge for treatment of a pulmonary embolism.

Experimental group: one patient was readmitted 3 days post-discharge for pain management and a wound infection.

\section{Discussion}

This study excluded patients with any air leak beyond $24 \mathrm{~h}$ after surgery, allowing us to focus on the sole criteria of chest drain output as a criterion for chest drain removal.

Table 3 Complications rate in both groups

\begin{tabular}{lll}
\hline Diagnosis & E Group (7/40) & $\begin{array}{l}\text { C Group } \\
(9 / 40)\end{array}$ \\
\hline Exacerbation COPD & 1 & 1 \\
Atrial fibrillation & 2 & 1 \\
Pneumonia & 2 & 4 \\
Delirium & 1 & 1 \\
Pneumonia and atrial fibrillation & 1 & 0 \\
Respiratory failure & 0 & 1 \\
Acute kidney failure & 0 & 1 \\
\hline
\end{tabular}

$C O P D$ chronic obstructive pulmonary disease; $E$ experimental; $C$ control
Chest drains are routinely placed into the pleural space following lobectomy by the vast majority of thoracic surgeons for safety (to prevent tension pneumothorax) and to have insight into possible complications, such as postoperative bleeding.

Chest drain removal favors better mobilization, reduced pain, and thereby shorter length of hospital stay-which all may potentially promote both lower complication rates and hospital costs, However there is poor consensus about the criteria for CDR when there is not an air leak.

The one prospective randomized trial used absolute thresholds of 150,300 , and $450 \mathrm{ml} / \mathrm{d}$ for CDR and found that $300 \mathrm{ml} / \mathrm{d}$ was the safe threshold [11].

Other randomized controlled studies (RCT) to answer different questions related to CDR after lung surgery used different thresholds for CDR. In one study [20] this threshold was established as $250 \mathrm{ml}$, in another [21] it was as $200 \mathrm{ml}$. A third study [9] puts up no upper limit prior to CDR, demonstrating, however, in nearly $10 \%$ of patients a need for immediate re-intervention, either by means of $\mathrm{CD}$ reinsertion or thoracentesis. In none of these studies was there any information about the patients after discharge (specifically if subsequent pleural intervention was required).

The aim of this RCT was to explore a threshold for CDR based on the patient's size or more specifically weight as a surrogate for size.

Obesity is a potential confounder because obese patients actually have a smaller pleural space than slim patients (the diaphragm is pushed up by the obese abdomen), but their weight alone would indicate a larger pleural space. The BMI would therefore theoretically be a better index than the patient's weight. However, height and weight are readily available, whereas in most hospital systems the BMI is either harder to find or needs to be calculated. In addition, $5 \mathrm{ml} /$ $\mathrm{kg}$ is very easy to calculate and can be done by most people without a calculator. Our priority was to have a simple, easy method that is highly reproducible. The average BMI of our patients was close to the German average for patients of this age [22] and is already on the cusp of being overweight (BMI between 25 and 30) with an average BMI of 25.2 (group E) and 24 (group C). In obese patients (BMI > 30), $20 \mathrm{~kg}$ can perhaps be taken off of their body weight prior to making this calculation if this is a concern [23].

The intra-pleural re-intervention rate was identical in both groups at one each. The power calculations for this study were based on CDD and it is entirely possible that this study was underpowered to address this issue in a robust manner.

We included over $10 \%$ more patients than our power calculations mandated ( $80 \mathrm{vs} 72$ ), so the conclusion of our study that weight-based CDR at $5 \mathrm{ml} / \mathrm{kg} / \mathrm{d}$ is beneficial in terms of CDD is both statistically and clinically highly significant as well as valid. 
We also have performed systematic lymph node dissection at all of the enrolled patients, only one appearing to have a benign disease afterward (lung abscess).

The LOS was 1.5 days shorter in the experimental group and this was a statistically significant finding. However, the study was not powered for this criteria (we would have needed 280 cases for this). Moreover, as part of the protocol the patients should have been discharged on the day following CDR, which had not occurred in all patients as per protocol. There are two principle reasons for these delays: in Germany the hospital loses money if the patient stays less than 4 days and some patients feel they need an extra day to feel ready to go home. If either of these events occurred, we did not exclude the patients from the study. Thus the LOS findings are less robust than the CDD findings. Nonetheless, in practice and in most publications CDR and LOS are usually highly correlated [24-26].

In conclusion, this study shows that CDR as soon as drainage is less than $5 \mathrm{ml} / \mathrm{kg}$ of body weight per day leads to reduction in CDD as well as in LOS. Further study may be necessary to confirm a non-inferiority of the new threshold regarding the need for intra-pleural re-intervention.

Acknowledgements Special thanks to our statistician Mrs. Ana Beslin for her support.

Author contributions DS assisted in conceptualization, data capture, formal analysis, methodology, administration, and writing of the manuscript. MD assisted in validation and writing (reviewing and editing) of the manuscript. TS provided resources. ER assisted in validation and writing (review and editing) of the manuscript. AM assisted in data capture, methodology, supervision, validation, and administration.

Funding Open Access funding enabled and organized by Projekt DEAL.

\section{Declarations}

Disclosures Davor Stamenovic has no conflicts of interest or financial ties to disclose. Michael Dusmet has no conflicts of interest or financial ties to disclose. Thomas Schneider has no conflicts of interest or financial ties to disclose. Eric Roessner has no conflicts of interest or financial ties to disclose. Antje Messerschmidt has no conflicts of interest or financial ties to disclose.

Open Access This article is licensed under a Creative Commons Attribution 4.0 International License, which permits use, sharing, adaptation, distribution and reproduction in any medium or format, as long as you give appropriate credit to the original author(s) and the source, provide a link to the Creative Commons licence, and indicate if changes were made. The images or other third party material in this article are included in the article's Creative Commons licence, unless indicated otherwise in a credit line to the material. If material is not included in the article's Creative Commons licence and your intended use is not permitted by statutory regulation or exceeds the permitted use, you will need to obtain permission directly from the copyright holder. To view a copy of this licence, visit http://creativecommons.org/licenses/by/4.0/.

\section{References}

1. Miserocchi G, Agostoni E (1971) Contents of the pleural space. J Appl Physiol 30:208-213

2. Negrini D, Moriondo A (2013) Pleural function and lymphatics. Acta Physiol 207:244-259

3. Miserocchi G (1997) Physiology and pathophysiology of pleural fluid turnover. Eur Respir J 10:219-225

4. Utter GH (2013) The rate of pleural fluid drainage as a criterion for the timing of chest tube removal: theoretical and practical considerations. Ann Thorac Surg 96:2262-2267

5. Miserocchi G, Venturoli D, Negrini D, Del Fabbro M (1993) Model of pleural fluid turnover. J Appl Physiol 75:1798-1806

6. Leckie WJ, Tothill P (1965) Albumin turnover in pleural effusions. Clin Sci 29:339-352

7. Stewart PB (1963) The rate of formation and lymphatic removal of fluid in pleural effusions. J Clin Investig 42:258-262

8. Koryllos A, Eggeling S, Schega O, Schweigert M, Leschber G (2020) Delphi-Konsens der Deutschen Gesellschaft für Thoraxchirurgie über das Management von Thoraxdrainagen. Zentralbl Chir 145:99-107

9. Holbek BL, Christensen M, Hansen HJ, Kehlet H, Petersen RH (2019) The effects of low suction on digital drainage devices after lobectomy using video-assisted thoracoscopic surgery: a randomized controlled trial $\dagger$. Eur J Cardiothorac Surg 55:673-681

10. Lijkendijk M, Neckelmann K, Licht PB (2018) External suction and fluid output in chest drains after lobectomy: a randomized clinical trial. Ann Thorac Surg 105:393-398

11. Xie HY, Xu K, Tang JX, Bian W, Ma HT, Zhao J, Bin N (2015) A prospective randomized, controlled trial deems a drainage of $300 \mathrm{ml} /$ day safe before removal of the last chest drain after videoassisted thoracoscopic surgery lobectomy. Interact Cardiovasc Thorac Surg 21:200-205

12. Fernandez FG, Falcoz PE, Kozower BD, Salati M, Wright CD, Brunelli A (2015) The society of thoracic surgeons and the European society of thoracic surgeons general thoracic surgery databases: joint standardization of variable definitions and terminology. Ann Thorac Surg 99:368-376

13. Mathis G (2010) Bildatlas der Lungen- und Pleurasonographie. Springer, Berlin, p 31

14. Metersky ML (2003) Is the lateral decubitus radiograph necessary for the management of a parapneumonic pleural effusion? Chest 124:1129-1132

15. Goecke W, Schwerk W (1990) Die Real -Time Sonographie in der Diagnostik von Pleuraergüssen. In: Gebhardt J, Hackelöer BJ, von Klinggräff G, Seitz K (eds), Ultraschall-diagnostik'89 Springer, Berlin, Heidelberg https://doi.org/10.1007/978-3-642-93467-4_ 98

16. Hassan M, Rizk R, Essam H, Abouelnour A (2017) Validation of equations for pleural effusion volume estimation by ultrasonography. J Ultrasound 20:267-271

17. R Core Team (2013) R: A language and environment for statistical computing. R Foundation for Statistical Computing, Vienna, Austria

18. Faul F, Erdfelder E, Lang AG, Buchner A (2007) G*Power 3: a flexible statistical power analysis program for the social, behavioral, and biomedical sciences. Behav Res Methods 39:175-191

19. Eldridge SM, Chan CL, Campbell MJ, Bond CM, Hopewell S, Thabane L, CONSORT et al (2010) Statement: extension to randomised pilot and feasibility trials. BMJ 2016:355

20. Chiappetta M, Lococo F, Nachira D, Ciavarella LP, Congedo MT, Porziella V, Meacci E, Margaritora S (2018) Digital devices improve chest tube management: results from a prospective randomized trial. Thorac Cardiovasc Surg 66:595-602 
21. Cui Z, Zhang Y, Xu C, Ding C, Chen J, Li C, Zhao J (2019) Comparison of the results of two chest tube managements during an enhanced recovery program after video-assisted thoracoscopic lobectomy: a randomized trial. Thoracic Cancer 10:1993-1999

22. Mensink G, Schienkiewitz A, Haftenberger M, Lampert T, Ziese T, Scheidt-Nave C (2013) Overweight and obesity in Germany. Robert Koch-Institut, Epidemiologie und Gesundheitsberichterstattung.

23. Moon K, Krems C, Heuer T, Roth A, Hoffmann I (2017) Predictors of BMI vary along the BMI range of German adults-results of the German national nutrition survey II. Obes Facts 10:38-49

24. Rena O, Massera F, Papalia E, Della Pona C, Robustellini M, Casadio C (2008) Surgical pleurodesis for Vanderschueren's stage III primary spontaneous pneumothorax. Eur Respir J 31:837-841

25. Dearden AS, Sammon PM, Matthew EF (2013) In patients undergoing video-assisted thoracic surgery for pleurodesis in primary spontaneous pneumothorax, how long should chest drains remain in place prior to safe removal and subsequent discharge from hospital? Interact Cardiovasc Thorac Surg 16:686-691

26. Zhou J, Lyu M, Chen N, Wang Z, Hai Y, Hao J, Liu L (2018) Digital chest drainage is better than traditional chest drainage following pulmonary surgery: a meta-analysis. Eur J Cardiothorac Surg 54:635-643

Publisher's Note Springer Nature remains neutral with regard to jurisdictional claims in published maps and institutional affiliations. 